\title{
Tecnologia de aplicação em café (Coffea arabica L.): efeito da assistência de ar com equipamento de acionamento manual
}

\section{Application technology in coffee (Coffea arabica L.): effect of air assistance with manually driven equipment}

\author{
João Miguel Francisco Ruas ${ }^{1}$; Gustavo Migliorini de Oliveira ${ }^{2 *}$; Augusto \\ Guilherme de Araújo ${ }^{3}$; Rafael Balan ${ }^{4}$; Leandro Ryiuiti Higashibara ${ }^{5}$; Otavio Jorge \\ Grigoli Abi Saab ${ }^{6}$
}

\section{Resumo}

Pequenos agricultores, nas diversas partes do Brasil, baseiam a aplicação de produtos fitossanitários exclusivamente em pulverizadores costais manuais. Como se trata de um equipamento bastante simples, poucos são os recursos disponíveis visando melhorar a qualidade da aplicação realizada. O objetivo deste trabalho foi estudar o efeito da assistência de ar, com acionamento manual, na deposição e porcentual de cobertura da calda aplicada em pulverização nas folhas de café, situadas interna e externamente. $\mathrm{O}$ equipamento constituiu-se de pulverizador costal pressurizado com $\mathrm{CO}_{2}$ acoplado a uma polvilhadora. $\mathrm{Na}$ saída de ar da polvilhadora foi adaptada uma mangueira para condução do fluxo de ar emitido junto ao bico de pulverização do pulverizador (XR 110-02, jato plano ampliado). Utilizou-se uma calda composta de água + cloreto de potássio + corante fluorescente. Os níveis de depósitos foram avaliados pela condutividade elétrica da solução de lavagem sobre as folhas e o percentual de cobertura através da luz ultravioleta emitida. A deposição foi maior nas folhas posicionadas externamente à planta, assim como a cobertura porcentual. Também a cobertura porcentual foi maior para a face adaxial das folhas de café. Não houve diferença quanto ao uso ou não da assistência de ar. Aperfeiçoamentos na geração e distribuição do ar em equipamentos de acionamento manual podem ser necessários, visando sua utilização para melhorias da aplicação de líquidos em pulverização.

Palavras-chave: Pulverizador, cobertura, cafeeiro

\begin{abstract}
Small farmers in various parts of Brazil, based the application of pesticides exclusively by a coastal manual sprayers. How it is simple equipment, there are few resources available to improve the quality of the application. The aim of this paper was to study the effect of air assistance, with manual override, in the deposition and percentage of coverage applied on the leaves of coffee plants, situated internally and externally. The equipment consisted of a sprayer coastal pressurized by $\mathrm{CO}_{2}$ attached to an air assistance equipment. At the exit of air assistance equipment a hose was adapted for driving the air flow
\end{abstract}

\footnotetext{
${ }^{1}$ Eng $^{\mathrm{o}} \mathrm{Agr}^{\mathrm{o}}$, M.e em Agronomia, Universidade Estadual de Londrina, UEL, Londrina, PR. E-mail: joaomiguelruas@hotmail.com

${ }^{2}$ Eng $^{\circ}$ Agr $^{\circ}$, Discente de Doutorado em Agronomia, UEL, Londrina, PR. E-mail: gmigliorini@hotmail.com

${ }^{3}$ Eng $^{\circ}$ Agrícola, Pesquisador Dr. Iapar, Londrina, PR. E-mail: agaraujo@iapar.br

${ }^{4}$ Eng $^{\text {o }}$ Agr $^{\circ}$, Uel, Londrina, PR. E-mail: rafaelbalan@hotmail.com

${ }^{5}$ Eng $^{\circ}$ Agr ${ }^{\circ}$, M.e em Agronomia, UEL, Londrina, PR. E-mail: cbtagro@gmail.com

${ }^{6}$ Eng $^{\mathrm{o}} \mathrm{Agr}^{\circ}$, Prof. Dr. Adjunto, Dept ${ }^{\circ}$ de Agronomia, UEL, Londrina, PR. E-mail: abisaab@uel.br

* Autor para correspondência
} 
emitted to the nozzle of the sprayer coastal (XR 110-02, expanded jet plane). It was used a solution of water + potassium chloride + fluorescent dye to the application. The levels of deposits were measured by electrical conductivity of the solution from the washing leaves and the percentage of coverage by ultraviolet light. The deposition was higher in leaves positioned externally to the plant, as well as the coverage percentage. The percentage of coverage was greater for the adaxial side of the coffee leaves. There was no difference for the use or not of the air assistance. Improvements in the generation and distribution of air in manually equipments may be necessary, in order to improve its use in the application of spray liquids.

Key words: Spray, coverage, coffee plants

\section{Introdução}

Entre as inúmeras técnicas de aplicação de produtos fitossanitários, as que se baseiam na pulverização hidráulica são as mais difundidas, graças à flexibilidade que oferecem em diferentes situações. Estes pulverizadores hidráulicos vão desde os mais simples, do tipo costal de acionamento manual utilizado em pequenas áreas, até os equipamentos mais sofisticados como os turbo-atomizadores e os pulverizadores de barra utilizados em áreas mais extensas.

Segundo a FAO (1998), pequenos agricultores nas diversas partes do Brasil baseiam a aplicação de produtos fitossanitários a partir de pulverizadores costais manuais nas mais variadas circunstâncias. Tal fato é confirmado por Ramos (2004) em áreas de agricultura familiar no Estado de São Paulo, em que o autor observou que os pulverizadores com alavanca manual representam em torno de $60 \%$ dos equipamentos utilizados. Vicente, Colho e Lopes Junior (1999) em estudo anterior semelhante, relatam ainda que tais pulverizadores costais não são projetados para atividades específicas, sendo normalmente de baixa capacidade operacional e não possuem contudo, um sistema eficiente de proteção contra a deriva.

A pressão proporcionada pelo ar comprimido no pistão destes equipamentos é o que fornece a energia para o deslocamento da calda até o bico de pulverização, representando assim um dos principais componentes que influenciam na qualidade e na segurança da aplicação. Sabe-se que a eficiência da aplicação está diretamente ligada ao ato de pulverizar a calda em questão, uma vez que após a saída do bico de pulverização as gotas têm seu movimento proporcional à sua massa e a sua velocidade inicial. Contudo, este processo não é muitas vezes suficiente para que as gotas penetrem em todos os extratos do dossel das culturas, tornando-se assim em um agravante para os tratos fitossanitários, haja vista que a maioria das doenças e pragas tem seu desenvolvimento inicial no interior das plantas. Principalmente, por exemplo, para fungicidas de contato, que requerem maior deposição e cobertura sobre as folhas nas plantas como um todo (GUPTA; DUC, 1996; PERGHER; GUBIANI; TONNETO, 1997).

Gil etal.(1996) citam ainda que a heterogeneidade do alvo (folhas e ramos), especialmente em plantas perenes, dificulta a aplicação no tocante a uniformidade de deposição e cobertura de tais gotas nas diferentes partes das plantas. Neste sentido, uma alternativa é a adoção da assistência de ar, ou seja, um fluxo de ar gerado mecanicamente para auxiliar no carregamento de tais gotas para o interior do dossel das plantas. Assim, a assistência de ar torna-se uma ferramenta bastante importante para a deposição da calda pulverizada sobre o alvo pretendido (KAUL et al., 2002; MATTHEWS, 2000; PERGHER; GUBIANI, 1995; VENEGAS; RAETANO; BAUER, 2003; SCUDELER et al., 2004).

Elonka e Parson (1976) definem fluxo como um fluido em movimento, incluindo tanto os líquidos como gases de uma forma geral. Portanto, de acordo com os princípios da mecânica dos fluidos 
(MESQUITA; GUIMARÃES; NEFUSSI, 1988), a vazão do fluxo é dependente da velocidade média do fluido e área transversal da saída do fluxo. O fluxo de ar no interior das culturas é, portanto, decorrente da velocidade aerodinâmica (RANDALL, 1971), da orientação e do número de saídas usadas para direcionar o ar (FURNESS; PINCZEWSKI, 1985; GOHLICH, 1985; PERGHER; GUBIANI; TONNETO, 1997; PEZZI; RONDELLI, 2000; PERGHER; GUBIANI, 1995).

No entanto, não há disponível atualmente no mercado um equipamento de baixo custo com assistência de ar junto à ponta de pulverização. Neste sentido, o objetivo deste trabalho foi estudar o efeito da assistência de ar, a partir de um equipamento com acionamento manual, na deposição e porcentual de cobertura da calda aplicada em pulverização na cultura do café.

\section{Material e Métodos}

O experimento foi conduzido na fazenda experimental da Universidade Estadual de Londrina, coordenadas geográficas $23^{\circ} 20^{\prime} 30^{\prime \prime} \mathrm{S} 51^{\circ} 12^{\prime} 35^{\prime \prime} \mathrm{O}$, altitude aproximada de $560 \mathrm{~m}$, clima classificado como Cfa, segundo KöPPen, e precipitação média anual de $1.600 \mathrm{~mm}$. A variedade de café utilizada foi Catuaí, implantada com espaçamento entrelinhas de 2,5 metros e 0,5 metros entre plantas. As plantas apresentavam altura aproximada de 2 metros.

$\mathrm{O}$ equipamento testado constituiu-se de pulverizador costal pressurizado com $\mathrm{CO}_{2}$ (lança única de pulverização) acoplado a uma ventoinha de acionamento manual, pertencente originalmente a uma polvilhadora da marca Jacto. Na saída de ar da polvilhadora foi adaptada uma mangueira para condução do fluxo de ar emitido junto ao bico de pulverização do pulverizador (XR 110-02, jato plano ampliado).

A partir deste equipamento, aplicou-se sobre a cultura do cafeeiro uma calda composta de solução de água + cloreto de potássio na concentração de
$3 \%+$ traçante fluorescente.

A aplicação foi efetuada apenas em um dos lados da cultura. A distância entre o bico de pulverização e as plantas de café foi de aproximadamente $0,5 \mathrm{~m}$. A pressão de trabalho estabelecida foi de 240 $\mathrm{kPa}$, gerando uma vazão de $0,74 \mathrm{~L} \cdot \mathrm{min}^{-1}$, que na velocidade do aplicador de $3 \mathrm{~km} \cdot \mathrm{h}^{-1}$ possibilitou uma taxa de aplicação de 59,2 L.ha ${ }^{-1}$. As condições climáticas no momento da aplicação foram aproximadamente $24{ }^{\circ} \mathrm{C}, 66 \%$ de umidade relativa do ar e $3 \mathrm{~km} . \mathrm{h}^{-1}$ de velocidade do vento.

Utilizou-se 10 parcelas, constituídas de uma única linha da cultura com 8 metros de comprimento, para cada tipo de aplicação - com e sem assistência de ar. Estudaram-se a partir de tal os níveis de depósito e os percentuais de cobertura da calda sobre as folhas. Neste sentido, adotou-se os delineamento experimental em esquema fatorial $2 \times 2$ para deposição, que envolviam os fatores assistência de ar (presente e ausente) e posição das folhas no dossel da planta (externa e interna). Já para o percentual de cobertura, o delineamento experimental idealizado foi de $2 \times 2 \times 2$, tendo como fatores a assistência de ar (presente e ausente), a posição das folhas no dossel da planta (externa e interna) e a face das folhas (abaxial e adaxial).

Após as aplicações, as folhas foram coletadas e armazenadas em sacos plásticos para posterior avaliação em laboratório. As folhas classificadas como da posição externa e interna situavam-se no $1^{\circ}$ e $4^{\circ}$ par de folhas, respectivamente. Para a avaliação de deposição da calda nas folhas, coletaram-se em cada parcela 3 amostras com 10 folhas cada. Nestes sacos plásticos foram adicionados $200 \mathrm{ml}$ de água destilada e deionizada para a mensuração da condutividade elétrica. Para a avaliação da porcentagem de cobertura da calda nas folhas, coletou-se 10 folhas em cada parcela, sendo armazenadas individualmente em sacos de papel. A metodologia foi baseada no trabalho Abi Saab e Antuniassi (1997). Posteriormente estas folhas foram iluminadas com luz ultravioleta, em ambiente 
escuro, e cada respectiva face foi fotografada com câmera digital. Às imagens geradas foram atribuídas notas visuais, em duplo cego, variando de 0 a 100 o percentual de cobertura.

Os dados de porcentagem de cobertura foram transformados para raiz quadrada de $x+0,5$ a fim de apresentarem normalidade. Assim, os resultados obtidos, tanto para deposição como porcentagem de cobertura, foram submetidos à análise de variância complementada pelo teste Tukey em nível de 5\% de probabilidade para comparação das médias.

\section{Resultados e Discussão}

Para a deposição não houve interação entre os fatores assistência de ar (presente ou ausente) e posição das folhas nas plantas (interna e externa). Os resultados, apresentados na Tabela 1, mostram que não houve diferença significativa entre a presença ou não da assistência de ar. A posição das folhas na planta, por sua vez, apresentou diferença significativa, apresentando maiores níveis de depósitos nas folhas posicionadas externamente ao dossel da planta.

Tabela 1. Condutividade elétrica $\left(u \mathrm{~S} . \mathrm{cm}^{-2}\right)$ da solução de lavagem das folhas de café para os fatores assistência de ar e posição das folhas no dossel das plantas de café.

\begin{tabular}{cc}
\hline \multicolumn{2}{c}{ Assistência de ar } \\
Presente & Ausente \\
$19,35 \mathrm{a}$ & $21,43 \mathrm{a}$ \\
\hline \multicolumn{2}{c}{ Posição das folhas } \\
Interna & Externa \\
$9,68 \mathrm{~b}$ & $30,85 \mathrm{a}$ \\
\hline CV $(\%)$ & 33,25 \\
\hline
\end{tabular}

* Médias seguidas de mesma letra nas linhas não diferem entre si pelo teste Tukey em nível de 5\% de probabilidade.

Fonte: Elaboração dos autores.

Quanto à porcentagem de cobertura, da mesma forma que para deposição, não houve nenhuma interação significativa entre os fatores assistência de ar, posição das folhas e face das folhas. Os resultados para a cobertura porcentual, apresentados na Tabela 2, evidenciam que não houve diferença significativa entre a presença ou não de assistência de ar. Já para a posição e face das folhas nas plantas, observou-se maior percentual de cobertura nas folhas externas e face adaxial, respectivamente.

Tabela 2. Porcentagem de cobertura para os fatores assistência de ar, posição em relação dossel das plantas e face das folhas de café.

\begin{tabular}{rr}
\hline \multicolumn{3}{c}{ Assistência de ar } \\
Presente & Ausente \\
$8,45 \mathrm{a}$ & $9,22 \mathrm{a}$ \\
\hline \multicolumn{3}{c}{ Posição das folhas } \\
Externa & Interna \\
$14,04 \mathrm{a}$ & $4,56 \mathrm{~b}$ \\
\hline & Face das folhas \\
Adaxial & Abaxial \\
$15,32 \mathrm{a}$ & $2,35 \mathrm{~b}$ \\
\hline $\mathrm{CV}(\%)$ & 26,71 \\
\hline
\end{tabular}

*Dados transformados para raiz quadrada de $\mathrm{x}+0,5$. ** Médias seguidas de mesma letra não diferem entre si pelo teste Tukey ao nível 5\% de probabilidade.

Fonte: Elaboração dos autores.

Apesar de não verificado o seu efeito neste trabalho, a assistência de ar é, sem dúvida, um importante auxiliar nas aplicações de produtos fitossanitários, seja em pulverizadores de barras para cultivos anuais ou em turbo-pulverizadores para culturas perenes. Matthews (2000) destaca que o uso apropriado de assistência de ar é exigido para melhorar a distribuição da calda e eficiência de deposição dos produtos fitossanitários aplicados nas diversas partes da planta. Estas premissas, além da possibilidade de redução dos índices de deriva, são assinaladas por diversos outros autores (VENEGAS; RAETANO; BAUER, 2003; SCUDELER et al., 2004; PERGHER; GUBIANI, 1995; KAUL et al., 2002; COOKE et al., 1990; BAUER; RAETANO, 2000; MATTHEWS, 2000; RAETANO, 2002).

A assistência de ar pode interferir tanto no deslocamento das gotas no espaço como também na movimentação das folhas das plantas, 
possibilitando assim uma maior penetração destas no dossel das culturas. Neste sentido, presume-se que a assistência de ar gerada por tal equipamento testado (acionamento manual) pode não ter sido suficiente para carregar as gotas e movimentar as folhas, e assim melhorar os níveis de deposição e cobertura na posição interna, bem como a cobertura na face abaxial das folhas, haja vista que estas apresentavam a face adaxial voltada diagonalmente ao deslocamento das gotas. Este argumento é embasado no trabalho de Ruas, Balan e Abi Saab (2011), que concluíram que a assistência de ar, gerada por um turbo-atomizador costal, promoveu melhor cobertura nas folhas situadas internamente e na parte mediana da planta de café.

Destaca-se ainda, que quanto maior a distância entre as pontas de pulverização e o alvo, menor a capacidade do ar em transportar as gotas e maior é a probabilidade de evaporação e deriva destas (RAMOS, 2004). Segundo Farooq et al. (2001) isso ocorre em virtude da redução da velocidade das gotas pulverizadas durante o seu deslocamento até o alvo, em função das forças físicas atuantes no processo.

No entanto, os níveis dos depósitos nas plantas alvo não dependem somente do equipamento utilizado, mas também da idade, do tipo da cultura, densidade de plantas, momento de aplicação e condição operacional durante a pulverização (COOKE et al., 1990). Neste sentido, o formato das plantas de café deste experimento contribuíram para a desuniformidade da aplicação nas diferentes posições das folhas, o que era previamente esperado. Isto se deve principalmente a proximidade das folhas externas à ponta de pulverização, além do número de folhas nos ramos que as continham, em torno de cinco pares de folhas com pequena distância entre si, o que promoveu uma importante barreira para com as folhas posicionadas internamente.

Outro ponto que merece destaque referese à taxa de aplicação, pois a velocidade de deslocamento do aplicador tem grande influência nesta e, conseqüentemente, na deposição da planta (SALYANI; WHITNEY, 1988; PERGHER; GUBIANI; TONNETO, 1997). A taxa de aplicação, de menos de 60 L.ha $^{-1}$, aliada à concentração de $3 \%$ de $\mathrm{KCl}$ na calda, podem ter sido determinantes para os baixos valores de condutividade elétrica obtidos (até $30 u \mathrm{~S}$ ) no experimento. E, assim, podem ter colaborado para que não houvesse maiores diferenças entre as posições das folhas quando do uso da assistência de ar. Ruas, Balan e Abi Saab (2011), por exemplo, utilizaram taxa de aplicação de 100 L.ha-1 de calda para avaliar o efeito da assistência de ar de um turbo-atomizador costal, verificando diferença significativa na cobertura porcentual em folhas de café. Balan et al. (2008), por sua vez, com a mesma metodologia, usando concentração de $5 \%$ de $\mathrm{KCl}$ e taxa de aplicação de até 400 L.ha ${ }^{-1} \mathrm{em}$ alvos artificiais, obtiveram valores de até $400 \mu \mathrm{S}$ nas leituras em condutivímetro.

Embora o equipamento testado não tenha apresentado influência significativa, adequações e novas condições e ambientes de estudo podem proporcionar melhores resultados. No equipamento utilizado, um melhor aproveitamento da energia utilizada pelo aplicador para mover a alavanca de acionamento manual, poderia promover melhorias no fluxo de ar. A corrente de ar dentro da cultura é afetada diretamente pela taxa de corrente de ar gerada e sua velocidade aerodinâmica (RANDALL, 1971), assim como pela orientação da corrente de ar e o número de saídas usadas para direcionar o ar e a calda pulverizada para a cultura (FURNESS; PINCZEWSKI, 1985; GOHLICH, 1985; PERGHER; GUBIANI; TONNETO, 1997; PEZZI; RONDELLI, 2000). Randall (1971), por exemplo, relata que para a mesma potência, um fluxo de ar mais alto com baixa velocidade aerodinâmica, propiciou melhor penetração da calda quanto ao percentual de cobertura das folhas de macieira, do que baixo volume de ar com velocidade mais alta.

Finalmente, estudos neste âmbito têm importância fundamental principalmente para os pequenos produtores que não tem acesso muitas 
vezes a tecnologias mais sofisticadas e modernas. Melhorias na deposição e cobertura das gotas nas partes internas das plantas podem melhorar substancialmente a eficácia da aplicação, como no caso de fungicidas de contato que requerem maior cobertura nas plantas como um todo Gupta e Duc (1996) e Pergher, Gubiani e Tonneto (1997).

\section{Conclusões}

Com base nos resultados observados concluíse que não houve diferença quanto ao uso, ou não, de ar com acionamento manual para deposição da calda e percentual de cobertura. Maiores níveis de deposição foram verificados nas folhas posicionadas externamente ao dossel da planta, enquanto que para o percentual de cobertura maiores valores foram encontrados nas folhas externas e faces adaxiais.

Aperfeiçoamentos na geração e distribuição do ar em equipamentos de acionamento manual são necessários, visando sua utilização para melhorias da aplicação de líquidos em pulverização.

\section{Referências}

ABI SAAB, O. J. G.; ANTUNIASSI, U. R. Avaliação do depósito de calda em um sistema de aplicação de defensivos utilizados em videiras. Energia na Agricultura, Botucatu, v. 12, n. 2, p. 1-11, 1997.

BALAN, M.; ABI SAAB, O.; SILVA, C.; RIO, A. Deposição da calda pulverizada por três pontas de pulverização sob diferentes condições meteorológicas. Semina: Ciências Agrárias, Londrina, v. 29, n. 2, p. $293-$ 298, 2008.

BAUER, F. C.; RAETANO, C. G. Assistência de ar na deposição e perdas de produtos fitossanitários em pulverizações na cultura da soja. Scientia Agricola, Piracicaba, v. 57, n. 2, p. 271-276, 2000.

COOKE, B. K.; HISLOP, E. C.; HERRINGTON, P. J.; WESTERN, N. M.; HUMPHERSON-JONES, F. Airassisted spraying of arable crops in relation to deposition, drift and pesticide performance. Crop Protection, Oxford, v. 9, n. 4, p. 303-311, 1990.

ELONKA, S. M.; PARSONS, A. R. Manual de instrumentação: sistemas de medição. São Paulo:
McGraw-Hill, v. 1, 1976. 254 p.

FOOD AND AGRICULTURE ORGANIZATION OF THE UNITED NATIONS - FAO technical standards: sprayer specifications and test procedures. In:

Agricultural pesticide sprayers. Roma: FAO, v. 2, 1998.

FAROOQ, M.; BALANCHANDAR, R.; WULFSOHN, D.; WOLF, T. M. Agricultural sprays in cross-flow and drift. Journal of Agricultural Engineering Research, London, v. 78, n. 4, p. 347-58, 2001.

FURNESS, G. O.; PINCZEWSKI, W. V. A comparison of the spray distribution obtained from sprayers with converging and diverging air jets with low volume air assisted spraying on citrus and grapevines. Journal of Agricultural Engineering Research, London, v. 32, n. 4, p. 291-310, 1985.

GIL, E.; BADIOLA, J.; ARMENGOL, E.; BERNAT, C. Design and verification of a moving equipment for orchard and vineyard sprayer calibration. In: INTERNATIONAL CONFERENCEONAGRICULTURALENGINEERING - AGENG, 1996, Madrid. Proceedings... Madrid: AgEng, 1996. 8 p. (Paper n. 96A-141).

GOHLICH, H. Deposition and penetration of sprays. In: SYMPOSIUM ON APPLICATION AND BIOLOGY, 28., 1985. Proceedings... British Crop Protection Council, Monogram, 1985. p. 173-182.

GUPTA, C. P.; DUC, T. X. Deposition studies of a handheld air-assisted electrostatic sprayer. Transactions of the $A S A E$, St. Joseph, v. 39, n. 5, p. 1633-1639, 1996.

KAUL, K.; GEBAUER, S.; RIETZ, S.; HENNING, H. Mechanisms of distribution of plant protection products sprayed in orchards. Nachrichtenblatt des Deutschen Pflanzenschutzdienstes, Stuttgart, v. 54, n. 5, p. 110-17, 2002.

MATTHEWS, G. A. A review of the use of air in atomization of sprays, dispersion of droplets down wind and collection on crop foliage. Aspects of Applied Biology, Wellesbourne, v. 57, p. 21-27, 2000.

MESQUITA, A. L. S.; GUIMARÃES, F. A.; NEFUSSI, N. Engenharia de ventilação industrial. In: completar nome dos editores. Verificação de sistemas de ventilação. São Paulo: CETESB, 1988. cap. 8, p. 401-420.

PERGHER, G.; GUBIANI, R. The effect of spray application rate and airflow rate on foliar deposition in a hedgerow vineyard. Journal of Agricultural Engineering Research, London, v. 61, n. 3, p. 205-216, 1995.

PERGHER, G.; GUBIANI, R.; TONNETO, G. Foliar deposition and pesticide losses from three air-assisted sprayers in a hedgerow vineyard. Crop Protection, Guildford, v. 16, n. 1, p. 25-33, 1997. 
PEZZI, F.; RONDELLI, V. The performance of an air-assisted sprayer operating in vines. Journal of Agricultural Engineering Research, London, v. 76, n. 4, p. 331-340, 2000.

RAETANO, C. G. Assistência de ar em pulverizadores de barra. Biológico, São Paulo, v. 64, n. 2, p. 221-225, 2002.

RAMOS, H. H. Mecanização: aliados na aplicação. Caderno Técnico Máquinas, Campinas, n. 27, p. 20-23, 2004.

RANDALL, J. M. The relationships between air volume and pressure on spray distribution in fruit trees. Journal of Agricultural Engineering Research, London, v. 16, p. 1-31, 1971.

RUAS, J. M.; BALAN, R.; ABI SAAB, O. J. G. Comparação da cobertura de pulverizadores costais de acionamento manual e motorizado. Semina: Ciências Agrárias, v. 32, n. 4, p. 1417-1422, 2011.
SALYANI, M.; WHITNEY, J. D. Evaluation of methodologies for field studies of spray deposition. Transactions oh the ASAE, St. Joseph, v. 31, n. 2, p. 390395, 1988.

SCUDELER, F.; RAETANO, C. G.; ARAUJO, D.; BAUER, F. C. Cobertura da pulverização e maturação de frutos do cafeeiro com ethephon em diferentes condições operacionais. Bragantia, Campinas, v. 63, n. 1, p. 129139, 2004.

VENEGAS, F.; RAETANO, C. G.; BAUER, F. C. Assistência de ar em barra de pulverização, deposição da calda e controle da pinta preta na cultura da batata. Summa Phytopathologica, Botucatu, v. 29, n. 4, p. 323329, 2003.

VICENTE, M. C. M.; COELHO, P. J.; LOPES JUNIOR, A. Programa de segurança e saúde do trabalhador rural: banco de dados. São Paulo: [s. n.], 1999. 1 CD-ROM. 
